\title{
Pediatric Neuro-oncology
}



Katrin Scheinemann - Eric Bouffet Editors

Pediatric

Neuro-oncology

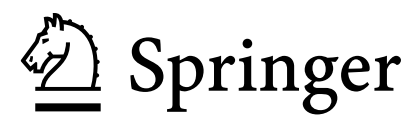




\section{Editors}

Katrin Scheinemann

Associate Professor of Pediatrics

Division of Hematology/Oncology

Department of Pediatrics

McMaster Children's Hospital

McMaster University

Hamilton, ON, Canada
Eric Bouffet

Garron Family Chair in Childhood

Cancer Research

Department of Hematology/Oncology

The Hospital for Sick Children

University of Toronto

Toronto, ON, Canada

ISBN 978-1-4939-1540-8

ISBN 978-1-4939-1541-5 (eBook)

DOI 10.1007/978-1-4939-1541-5

Springer New York Heidelberg Dordrecht London

Library of Congress Control Number: 2014954085

(C) Springer Science+Business Media New York 2015

This work is subject to copyright. All rights are reserved by the Publisher, whether the whole or part of the material is concerned, specifically the rights of translation, reprinting, reuse of illustrations, recitation, broadcasting, reproduction on microfilms or in any other physical way, and transmission or information storage and retrieval, electronic adaptation, computer software, or by similar or dissimilar methodology now known or hereafter developed. Exempted from this legal reservation are brief excerpts in connection with reviews or scholarly analysis or material supplied specifically for the purpose of being entered and executed on a computer system, for exclusive use by the purchaser of the work. Duplication of this publication or parts thereof is permitted only under the provisions of the Copyright Law of the Publisher's location, in its current version, and permission for use must always be obtained from Springer. Permissions for use may be obtained through RightsLink at the Copyright Clearance Center. Violations are liable to prosecution under the respective Copyright Law.

The use of general descriptive names, registered names, trademarks, service marks, etc. in this publication does not imply, even in the absence of a specific statement, that such names are exempt from the relevant protective laws and regulations and therefore free for general use.

While the advice and information in this book are believed to be true and accurate at the date of publication, neither the authors nor the editors nor the publisher can accept any legal responsibility for any errors or omissions that may be made. The publisher makes no warranty, express or implied, with respect to the material contained herein.

Printed on acid-free paper

Springer is part of Springer Science+Business Media (www.springer.com) 
To our patients and their families who teach us the essence of life every day:

Do not look back in the past or plan for the future; live life to the fullest today! 



\section{Foreword}

Pediatric Neuro-Oncology is undergoing a rapid transformation within all of its key disciplines. Rapid advances in our understanding in the biology of medulloblastoma, pontine glioma, low-grade and high-grade glioma, ependymoma, embryonal tumors abundant neuropil and true rosettes (ETANER), and atypical rhabdoid teratoid tumor (ATRT) have ushered in a new era of clinical and molecular diagnostics. Modern treatment approaches are being introduced into the clinic based on this advanced understanding of tumor biology, and current treatment approaches are being modified to the clinical and biological risk so as to reduce the long-term toxicity for patients with good prognosis.

Newer imaging techniques along with availability of intraoperative MRI have facilitated surgical resections that one could only have wished for a few decades ago. Sophistication in radiation treatment planning and the gradual availability of proton beam radiation therapy hold the promise of decreased long-term morbidity in children treated with focal radiation therapy.

A better understanding of the pathophysiology of the damage to the developing brain caused by current therapies has allowed neuropsychologists to initiate intervention studies that seek to mitigate the damage and provide the children with coping mechanisms.

Emerging interest in studying the quality of life and symptom burden in this patient population has led to refined end-of-life care. The editors, Drs. Scheinemann and Bouffet, and their Canadian team of coauthors have done an outstanding job of presenting this complex information in a lucid manner-this book is a must-read for the global community of aspiring students and neuro-oncology practitioners.

Amar Gajjar, MD

Member, St. Jude Faculty, Scott and Tracie Hamilton Endowed Chair in Brain Tumor Research Co-Chair, Department of Oncology Interim Chair, Department of Pediatric Medicine Director, Neuro-Oncology Division Co-Leader, Neurobiology \& Brain Tumor Program

St. Jude Children's Research Hospital Memphis, TN, USA 



\section{Preface}

Over the past decades the numbers of long-term survivors of pediatric brain tumors have increased. It was quickly recognized that this was influenced by modified multimodal treatment for which a multidisciplinary team was needed. In lots of large pediatric oncology units, specialized teams focused on pediatric neuro-oncology were created to take care of the complexity of these patients. Survivorship of this group also comes with significant longterm sequelae which have to be taken care of.

The purpose of this book is to provide a comprehensive practical, clinical, and research overview for pediatric neuro-oncology. It also emphasizes the role of national study groups as this book is authored by the members of the Canadian Pediatric Brain Tumor Consortium that involves many disciplines.

We would like to thank our chapter authors for their ideas, time, and knowledge devoted for this project.

We hope that this textbook will provide answers and guidance for healthcare professionals in this complex and challenging however fascinating field.

Hamilton, ON, Canada

Katrin Scheinemann

Toronto, ON, Canada

Eric Bouffet 



\section{Contents}

1 Introduction

Katrin Scheinemann and Eric Bouffet

2 Presentation of Central Nervous System Tumors

Ali Amid, Daniel L. Keene, and Donna L. Johnston

3 Epidemiology of Central Nervous System Tumors.

Daniel L. Keene and Donna L. Johnston

4 Pediatric Neuroimaging

Nina Rodrigues Stein and Luciana Torres Ribeiro

5 Neurosurgery.

Sami Obaid, Pierre-Olivier Champagne, Claude Mercier, and Louis Crevier

6 Histopathological Features of Common Pediatric Brain Tumors

Phedias Diamandis, Alaa Alkhotani, Jennifer A. Chan, and Cynthia E. Hawkins

7 Basic Science of Pediatric Brain Tumors

Stephen C. Mack, Vijay Ramaswamy, Xin Wang, Marc Remke, Patrick Sin-Chan, Tiffany Sin Yu Chan,

Kelsey C. Bertrand, Diana Merino, Kory Zayne, Annie Huang, and Michael D. Taylor

8 Cancer Predisposition in Children with Brain Tumors

Uri Tabori, Anne-Marie Laberge, Benjamin Ellezam, and Anne-Sophie Carret

9 Low Grade Glioma

Katrin Scheinemann and Juliette Hukin

10 High-Grade Glioma

Magimairajan Issai Vanan, Vivek Mehta, and David D. Eisenstat

11 Diffuse Intrinsic Pontine Glioma

Magimairajan Issai Vanan, Vivek Mehta, and David D. Eisenstat 
12 Embryonal Brain Tumors

Tiffany Sin Yu Chan, Xin Wang, Tara Spence,

Michael D. Taylor, and Annie Huang

13 Ependymoma

Juliette Hukin, John-Paul Kilday, and Uri Tabori

14 Central Nervous System Germ Cell Tumors (CNS-GCT)

Ute Bartels and Ash Singhal

15 Atypical Teratoid Rhabdoid Tumors

Lucie Lafay-Cousin, Douglas R. Strother, Jennifer A. Chan, Jonathon Torchia, and Annie Huang

16 Craniopharyngioma

David Phillips, Patrick J. McDonald, and Ute Bartels

17 Rare Tumours of the Central Nervous System in Children..

Adam J. Fleming

18 Radiotherapy

Anne-Marie Charpentier, Carolyn Freeman,

David Roberge, and Pierre Rousseau

19 Chemotherapy

Joan Lee and Donna L. Johnston

20 Targeted Therapies in Paediatric Brain Tumours

John-Paul Kilday, Nada Jabado, and Eric Bouffet

21 High-Dose Chemotherapy/Stem Cell

Transplantation (HDSCT)

Victor Anthony Lewis and Shahrad Rod Rassekh

22 Supportive Care

Beverly A. Wilson, Karina L. Black, and Samina Afzal

23 Long-Term Sequelae

Sebastien Perreault and Anne-Sophie Carret

24 Neuropsychological Outcomes in Pediatric

Brain Tumor Survivors.

Laura Janzen, Donald Mabbott, and Sharon L. Guger

25 Quality of Life

Annie-Jade Pépin, Anne-Sophie Carret, and Serge Sultan

26 Palliative Care for Children with Brain Tumors

Lisa Pearlman, Shayna Zelcer, and Donna L. Johnston

27 Pediatric Neuro-Oncology in Countries with Limited Resources

Eric Bouffet, Nisreen Amayiri, Adriana Fonseca, and Katrin Scheinemann 


\section{Contributors}

Samina Afzal, MBBS, FCPS, FRCPCH (UK) Department of Pediatrics, Division of Oncology, IWK Health Centre, Halifax, NS, Canada

Alaa Alkhotani, MD Department of Laboratory Medicine and Pathobiology, University of Toronto, Toronto, ON, Canada

Nisreen Amayiri, MD Department of Pediatrics, King Hussein Cancer Center, Amman, Jordan

Ali Amid, MD Department of Pediatrics, Division of Hematology/Oncology, Children's Hospital of Eastern Ontario, University of Ottawa, Ottawa, ON, Canada

Ute Bartels, MD Department of Hematology/Oncology, The Hospital for Sick Children, Toronto, ON, Canada

Kelsey C. Bertrand, MD Department of Developmental and Stem Cell Biology, The Hospital for Sick Children, Toronto, ON, Canada

Karina L. Black, BScN, MN, NP Pediatric Oncology Program, Stollery Children's Hospital, Edmonton, AB, Canada

Eric Bouffet, MD Department of Hematology/Oncology, The Hospital for Sick Children, University of Toronto, Toronto, ON, Canada

Anne-Sophie Carret, MD Department of Pediatrics, Division of Hematology/Oncology, CHU Sainte-Justine/Université de Montréal, Montréal, QC, Canada

Pierre-Olivier Champagne, MD Department of Neurosurgery, Hôpital Notre-Dame/Université de Montréal, Montréal, QC, Canada

Jennifer A. Chan, MD Department of Pathology \& Laboratory Medicine, University of Calgary, Calgary, AB, Canada

Tiffany Sin Yu Chan, BSc Department of Cell Biology/Arthur and Sonia Labatt Brain Tumor Research Centre, The Hospital for Sick Children, Toronto, ON, Canada

Anne-Marie Charpentier, MD, FRCPC Department of Radiation Oncology, CHUM Notre-Dame Hospital, Montréal, QC, Canada 
Louis Crevier, MD, MSc, FRCSC Department of Neurosurgery, Centre Hospitalier Universitaire de Sainte-Justine, Montréal, QC, Canada

Phedias Diamandis, MD, PhD Department of Laboratory Medicine and Pathobiology, University of Toronto, Toronto, ON, Canada

David D. Eisenstat, MD, MA, FRCPC Department of Pediatrics, Medical Genetics and Oncology, Stollery Children's Hospital, University of Alberta, Edmonton Clinic Health Academy, Edmonton, AB, Canada

Benjamin Ellezam, MD, PhD Department of Pathology, CHU SainteJustine, Université de Montréal, Montréal, QC, Canada

Adam J. Fleming, MASc, MD, FRCP(C) Division of Hematology/ Oncology, The Montreal Children's Hospital (McGill University Health Center), Montréal, QC, Canada

Adriana Fonseca, MD Department of Pediatrics, McMaster Children's Hospital, Hamilton, ON, Canada

Carolyn Freeman, MBBS, FRCPC, FASTRO Department of Radiation Oncology, McGill University Health Centre, Montréal, QC, Canada

Sharon L. Guger, PhD Department of Psychology, The Hospital for Sick Children, Toronto, ON, Canada

Cynthia E. Hawkins, MD, PhD, FRCPC Department of Paediatric Laboratory Medicine, The Hospital for Sick Children, Toronto, ON, Canada

Annie Huang, MD, PhD Department of Cell Biology, The Hospital for Sick Children, Toronto, ON, Canada

Juliette Hukin, MBBS, FRCPC Division of Neurology and Oncology, British Columbia Children's Hospital, Vancouver, BC, Canada

Nada Jabado, MD, PhD Department of Pediatrics, McGill University Health Center/McGill University, Montréal, QC, Canada

Laura Janzen, PhD Department of Psychology, The Hospital for Sick Children, Toronto, ON, Canada

Donna L. Johnston, MD, FRCPC, FAAP Department of Pediatrics, Division of Hematology/Oncology, Children's Hospital of Eastern Ontario, University of Ottawa, Ottawa, ON, Canada

Daniel L. Keene, BSc (Med), MD, MA, FRCPC Department of Pediatrics, Children's Hospital of Eastern Ontario, Ottawa, ON, Canada

John-Paul Kilday, MBChB, MRCPCH, PhD Pediatric Brain Tumor Program, Department of Hematology/Oncology, Hospital for Sick Children, Toronto, ON, Canada

Anne-Marie Laberge, MD, PhD Medical Genetics Division, Department of Pediatrics, Université de Montréal, Montréal, QC, Canada

Lucie Lafay-Cousin, MD, MSc Department of Pediatrics, Division of Oncology, Alberta Children's Hospital, Calgary, AB, Canada 
Joan Lee, BScPhm Department of Pediatrics, Division of Hematology/ Oncology, McMaster Children's Hospital, Hamilton, ON, Canada

Victor Anthony Lewis, MD Department of Pediatrics, Alberta Children's Hospital, Calgary, AB, Canada

Donald Mabbott, BA, MA, PhD Department of Psychology, The Hospital for Sick Children, Toronto, ON, Canada

Stephen C. Mack, BSc Department of Developmental and Stem Cell Biology, The Hospital for Sick Children, Toronto, ON, Canada

Patrick J. McDonald, MD, MHSc, FRCSC Department of Neurosurgery and Pediatrics, Winnipeg Children's Hospital, University of Manitoba, Winnipeg, MB, Canada

Vivek Mehta, MD, MSc Division of Neurosurgery, Department of Surgery, Stollery Children's Hospital/University of Alberta, Edmonton, AB, Canada

Claude Mercier, MD, FRCSC Department of Neurosurgery, Centre Hospitalier Universitaire de Sainte-Justine, Montréal, QC, Canada

Diana Merino, BSc Department of Genetics and Genome Biology, The Hospital for Sick Children, Toronto, ON, Canada

Sami Obaid, MDCM Department of Neurosurgery, Centre Hospitalier Universitaire de Sainte-Justine, Montréal, QC, Canada

Lisa Pearlman, RN(EC) NP-P, BAMN, ACNP Department of Paediatric Symptom Management \& Supportive Care, Children's Hospital London Health Sciences Centre, Victoria Hospital, London, ON, Canada

Annie-Jade Pépin, PhD Department of Hematology/Oncology, CHU SainteJustine, Université de Montréal, Montréal, QC, Canada

Sebastien Perreault, MD Division of Child Neurology, Lucile Packard Children's Hospital, Stanford University, Palo Alto, CA, USA

Department of Pediatrics, Division of Neurology, CHU Sainte-Justine/ Université de Montréal, Montréal, QC, Canada

David Phillips, BASC (Eng), MScSS, MD, FRCSC Department of Neurosurgery, University of Manitoba, Winnipeg, MB, Canada

Vijay Ramaswamy, MD, FRCPC Division of Neurosurgery, Hospital for Sick Children, Toronto, ON, Canada

Shahrad Rod Rassekh, MD, MHSc Division of Pediatric Oncology, Department of Pediatrics, British Columbia's Children's Hospital, Vancouver, BC, Canada

Marc Remke, MD Department of Laboratory Medicine and Pathobiology, The Hospital for Sick Children, Toronto, ON, Canada

Luciana Torres Ribeiro, MD, MSc, PhD, FRCPC, ABR Department of Radiology, Hamilton General Hospital, McMaster's Children's Hospital, McMaster University, Hamilton, ON, Canada 
David Roberge, MD, FRCPC Department of Radiation Oncology, CHUM Notre-Dame Hospital, Montréal, QC, Canada

Pierre Rousseau, MD Department of Radiation Oncology, CHUM NotreDame Hospital, Montréal, QC, Canada

Katrin Scheinemann, MD Division of Hematology/Oncology, Department of Pediatrics, McMaster Children's Hospital, McMaster University, Hamilton, ON, Canada

Patrick Sin-Chan, MSc Arthur and Sonia Labatt Brain Tumour Research Centre, The Hospital for Sick Children, Toronto, ON, Canada

Ash Singhal, MD, FRCS(C) Department of Pediatric Neurosurgery, University of British Columbia, BC Children's Hospital, Vancouver, BC, Canada

Tara Spence, MSc Department of Cell Biology, The Hospital for Sick Children, Toronto, ON, Canada

Nina Rodrigues Stein, MD, MSc Department of Diagnostic Imaging, McMaster University Hamilton Health Sciences, Hamilton, ON, Canada

Douglas R. Strother, MD Departments of Oncology and Pediatrics, Section of Oncology and Blood and Marrow Transplant, Cumming School of Medicine and Alberta Children's Hospital, Calgary, AB, Canada

Serge Sultan, PhD Department of Hematology/Oncology, CHU SainteJustine, Université de Montréal, Montréal, QC, Canada

Uri Tabori, MD Department of Hematology/Oncology, The Hospital for Sick Children/Institute of Medical Sciences, University of Toronto/The Arthur and Sonia Labatt Brain Tumour Research Centre, Toronto, ON, Canada

Michael D. Taylor, MD Department of Developmental and Stem Cell Biology, The Hospital for Sick Children, Toronto, ON, Canada

Jonathon Torchia, MSc Department of Lab Medicine and Pathobiology, Labatt's Brain Tumour Centre, SickKids, University of Toronto, Toronto, ON, Canada

Magimairajan Issai Vanan, MD, MPH, FAAP Departments of Pediatrics and Child Health, Oncology and Cell Biology, Cancer Care Manitoba, Manitoba Institute of Cell Biology, University of Manitoba, Winnipeg, MB, Canada

Xin Wang, BHSc (Hon) Department of Developmental and Stem Cell Biology, The Hospital for Sick Children, Toronto, ON, Canada

Beverly A. Wilson. BMSc, MD, FRCPSC Department of Pediatrics, Division of Hematology/Oncology, University of Alberta, Edmonton, AB, Canada

Kory Zayne, BSc Department of Developmental and Stem Cell Biology, The Hospital for Sick Children, Toronto, ON, Canada

Shayna Zelcer, BSc, MD Department of Pediatrics, Children's Hospital, London Health Sciences Center, London, ON, Canada 